\title{
Author Correction: Graphene active sensor arrays for long-term and wireless mapping of wide frequency band epicortical brain activity
}

\author{
R. Garcia-Cortadella, G. Schwesig, C. Jeschke, X. Illa (D), Anna L. Gray, S. Savage, E. Stamatidou, I. Schiessl (D), \\ E. Masvidal-Codina, K. Kostarelos (1), A. Guimerà-Brunet, A. Sirota \& J. A. Garrido (D)
}

Correction to: Nature Communications https://doi.org/10.1038/s41467-020-20546-w; published online 11 Jan 2021.

The original version of this article included an incorrectly named funding source "Graphene Flagship", and omitted the names of GrapheneCore 2 and GrapheneCore3 as funding sources, from the Acknowledgements. This has now been corrected in both the PDF and HTML versions of the Article.

The Acknowledgements now reads “This work has been funded by the European Union's Horizon 2020 research and innovation program under Grant Agreement No. 732032 (BrainCom) and Graphene Flagship Grant Agreements No. 785219 (GrapheneCore2) and 881603 (GrapheneCore3). The ICN2 is supported by the Severo Ochoa Centres of Excellence program, funded by the Spanish Research Agency (AEI, grant no. SEV-2017-0706), and by the CERCA Program/Generalitat de Catalunya. R.G.C. is supported by the International Ph.D Program La Caixa-Severo Ochoa (Programa Internacional de Becas "la Caixa"-Severo Ochoa). This work has made use of the Spanish ICTS Network MICRONANOFABS partially supported by MICINN and the ICTS "NANBIOSIS", more specifically by the Micro-NanoTechnology Unit of the CIBER in Bioengineering, Biomaterials, and Nanomedicine (CIBER-BBN) at the IMBCNM. This work is within the project FIS2017-85787-R funded by the "Ministerio de Ciencia, Innovación y Universidades" of Spain, the "Agencia Estatal de Investigación (AEI)", and the "Fondo Europeo de Desarrollo Regional (FEDER/UE)". A.S. and G.S. were also supported by Bundesministerium für Bildung und Forschung [grant number 01GQ0440]. R.G.C. acknowledges that this work has been done in the framework of the Ph.D in Electrical and Telecommunication Engineering at the Universitat Autònoma de Barcelona. We thank Eduardo Blanco Hernández for assistance with the preprocessing of the motion tracking data.

Published online: 30 April 2021

\footnotetext{
(c) (i) Open Access This article is licensed under a Creative Commons Attribution 4.0 International License, which permits use, sharing, adaptation, distribution and reproduction in any medium or format, as long as you give appropriate credit to the original author(s) and the source, provide a link to the Creative Commons license, and indicate if changes were made. The images or other third party material in this article are included in the article's Creative Commons license, unless indicated otherwise in a credit line to the material. If material is not included in the article's Creative Commons license and your intended use is not permitted by statutory regulation or exceeds the permitted use, you will need to obtain permission directly from the copyright holder. To view a copy of this license, visit http://creativecommons.org/licenses/by/4.0/.
}

(c) The Author(s) 2021 